\title{
The Analytic Coarse-Mesh Finite Difference Method for Multigroup and Multidimensional Diffusion Calculations
}

\author{
José M. Aragonés, * Carol Ahnert, and Nuria García-Herranz \\ Universidad Politécnica de Madrid, Department of Nuclear Engineering, \\ c/ José G. Abascal, 2; 28006 Madrid, Spain \\ Received September 26, 2005 \\ Accepted October 7, 2006
}

\begin{abstract}
In this work we develop and demonstrate the analytic coarse-mesh finite difference (ACMFD) method for multigroup — with any number of groups - and multidimensional diffusion calculations of eigenvalue and external source problems. The first step in this method is to reduce the coupled system of the $G$ multigroup diffusion equations, inside any homogenized region (or node) of any size, to the G independent modal equations in the real or complex eigenspace of the $G \times G$ multigroup matrix. The mathematical and numerical analysis of this step is discussed for several reactor media and number of groups.

As a second step, we discuss the analytical solutions in the general (complex) modal eigenspace for one-dimensional plane geometry, deriving the generalized Chao's relation among the surface fluxes and the net currents, at a given interface, and the node-average fluxes, essential in the ACMFD method. We also introduce here the treatment of heterogeneous nodes, through modal interface flux discontinuity factors, and show the analytical and numerical application to core-reflector problems, for a single infinite reflector and for reflectors with two layers of different materials.

Then, we address the general multidimensional case, with rectangular $X-Y-Z$ geometry considered, showing the equivalency of the methods of transverse integration and incomplete expansion of the multidimensional fluxes, in the real or complex modal eigenspace of the multigroup matrix. A nonlinear iteration scheme is implemented to solve the multigroup multidimensional nodal problem, which has shown a fast and robust convergence in proof-of-principle numerical applications to realistic pressurized water reactor cores, with heterogeneous fuel assemblies and reflectors.
\end{abstract}

\section{INTRODUCTION}

The analytic coarse-mesh finite difference (ACMFD) method was formally introduced by $\mathrm{Chao}^{1}$ for the onegroup one-dimensional (1-D) (plane) diffusion equation with transverse leakage as an external source. The generalization to multigroups and multidimensional problems was then just briefly outlined, with more details given for implementation in two-dimensional (2-D) hexagonal geometry. Recently, $\mathrm{Chao}^{2}$ added some quite significant comments on the analytic base function expansion for multidimensional nodal diffusion.

In the context of our own code system for pressurized water reactor (PWR) core analysis [SEANAP (Ref. 3)], we did develop and implement a new methodology that accounts for the linear and nonlinear depen-

*E-mail: arago@din.upm.es dences of the two-group equivalent diffusion parameters on flux-spectrum and burnup intranodal distributions. ${ }^{4}$ This methodology was later extended to implement Chao's ACMFD method, rigorously derived for homogeneous nodes in two groups and generalized by us for heterogeneous nodes,, 56 introducing heterogeneity factors that are nonlinearly dependent on the intranodal flux gradients.

The performance demonstrated in the 2-D $(X-Y)$ and two-group implementation of this method ${ }^{5}$ was excellent, with a very fast convergence of the nonlinear iterations (on nodal two-group constants, heterogeneity factors, and transverse leakage) and high accuracy as compared with fine-mesh calculations, while saving the computing time spent to solve the two-node problems required in the standard nodal expansion methods (NEMs).

The key feature of the ACMFD method is that the analytic solution of the diffusion equation within 
homogeneous regions leads to a linear relation among the node-average fluxes and the interface integrated fluxes and currents at any node face. In the absence of spacedependent external sources or transverse leakages from multidimensional problems (i.e., flat source or transverse leakage), the coefficients of this linear relation are the same for any node face. For realistic source, multidimensional, and heterogeneous problems, the coefficients need to be nonlinearly iterated and vary for each node face, becoming directionally dependent.

For multigroup problems these relations are scalar only in the modal space of the eigenvectors associated with each eigenvalue of the matrix of the multigroup equations, becoming matrix-vector relations in the physical space of the group fluxes and currents, resulting in the "coupling" of all groups. By equating the physical (continuous) fluxes and currents at the nodal interfaces between any two neighbor nodes, an ACMFD matrix relation is obtained, which gives the group interface current vector in terms of the difference of the products of two different "effective-diffusion" matrices by the vectors of the nodal average group fluxes at both neighbor nodes. Hence, Fick's Law not only is corrected in the diffusion coefficient and in the scalar flux gradient for coarse-mesh nodes but also results in a nonsymmetric matrix relation that couples the node-average fluxes of all groups at both neighbor nodes to yield the vector for all groups of the net currents at the interface. The sevendiagonal symmetric linear system that results from the three-dimensional (3-D) fine-mesh finite difference diffusion scheme for each group (with "external" source iteration) becomes a seven-block $(G \times G$ blocks $)$ diagonal nonsymmetric linear system for all the $G$ coupled groups in 3-D nodes, which are amenable to Krylov space solvers, such as GMRES (Ref. 6).

Thus, in summary, the ACMFD scheme is not an ad hoc approximation or discretization but the one that comes out explicitly from the analytic solution of the multigroup diffusion equations. The resulting generalized finite difference matrix-vector relations should be regarded as a high-order scheme since it includes the effects of the intranodal flux shape and spectral analytic variation. Although this is only rigorous for homogeneous nodes, we showed in our previous work ${ }^{5,6}$ that by introducing interface-dependent scalar heterogeneity factors for each node type, precalculated by single-assembly lattice calculations and a few parametrical colorsets, the ACMFD method can be effectively and accurately implemented for actual and quite heterogeneous operating reactors.

\section{THE ANALYTIC MULTIGROUP DIFFUSION THEORY}

The basis for the analytic solution of the multigroup diffusion equations within homogeneous regions lies in the elimination of the nondiagonal group-to-group coupling terms, due to scattering and fission, by the transformation of the physical space of group fluxes into the modal space of the complete base of eigenvectors of the multigroup matrix. This has been known in linear algebra for a long time - as found in early quantum mechanics and iterative analysis books ${ }^{7,8}$ - and is now general basic knowledge in books ${ }^{9,10}$ and guides. ${ }^{11}$ In reactor analysis, the analytic multigroup diffusion solutions were popularized by Henry ${ }^{12}$ and applied by his students in many $\mathrm{MS}$ and $\mathrm{PhD}$ works. We also applied them in the mid-1980s as the basis of our synthetic lineardiscontinuous coarse-mesh finite difference method. ${ }^{13,14}$ More recently, Chao introduced the general ACMFD method $^{1}$ and reviewed it in comparison with other previous approximations..$^{15}$ The decoupled modal space approach by a similarity transformation has also been extensively developed and successfully applied by Noh and $\mathrm{Cho}^{16}$; Cho, Kim, and Park ${ }^{17}$; and Woo, Cho, and $\mathrm{Noh}^{18}$ in their analytic function expansion nodal method for multigroup and multidimensional reactor diffusion analysis.

In this work we address another main problem found in the generalization of the ACMFD method for any number of neutron diffusion groups, as is the frequent appearance of complex eigenvalues and eigenfunctions, even for as few as three groups, depending on the group cutoffs. We solved that first by working on a complex modal space and deriving Chao's coefficients in that space, using complex exponential functions. Then, the transformation back to the physical group space yields real matrixvector relations, which can be applied as in the twogroup diffusion equations, where the eigenvalues are usually real. A summary of this complex algebra follows.

\section{II.A. Diagonalizing the Multigroup Diffusion Matrix: Uncoupled Modal Equations}

Let $\boldsymbol{A}$ be the matrix of the (coupled) system of $G$ equations, where $G$ is the number of neutron groups, in multigroup neutron diffusion theory inside a homogenized region (constant coefficients):

$$
\nabla^{2}|\boldsymbol{\phi}(\boldsymbol{r})\rangle-\boldsymbol{A}|\boldsymbol{\phi}(\boldsymbol{r})\rangle=-\boldsymbol{D}^{-1}|\boldsymbol{S}(\boldsymbol{r})\rangle,
$$

where the (column) vectors of group fluxes and external source are enclosed in the bracket notation. The matrix $\boldsymbol{A}$ is a real and square $G \times G$ matrix, which includes

1. in its diagonal terms the macroscopic group removal cross section (absorption + outscatter)

2. in its lower | upper triangular terms the in-down | upscatter cross sections from other groups, with minus sign

3. in its first rows the nu-fission cross sections, multiplied by the fission spectrum in the top groups and divided by the system (whole reactor) $k_{\text {eff }}$ eigenvalue, if applicable, with minus sign 
4. all rows being divided by the diffusion coefficient of the corresponding group.

The multigroup matrix $\boldsymbol{A}$ is nonsymmetric, nonnormal, and only partially diagonally dominant, with all diagonal terms positive and large and all nondiagonal terms negative or zero. In general, $\boldsymbol{A}$ is a diagonalizable $\boldsymbol{Z}$ matrix and in many cases an $\boldsymbol{M}$ matrix ${ }^{7-10^{\mathrm{a}}}$ : The spectrum of $\boldsymbol{A}$ includes only distinct and simple eigenvalues. The dominant smallest eigenvalue (fundamental mode) is real and can be negative or positive but close to zero, with all the associated eigenvectors of equal sign. All the other eigenvalues have a positive real part of increasing magnitude, with eigenvectors that can change sign. All these eigenvectors form a complete set. For nonmultiplicative or multiplicative subcritical media $\left(k_{\infty}<k_{\text {eff }}\right)$, all eigenvalues are positive, and $\boldsymbol{A}$ is an $\boldsymbol{M}$ matrix and thus nonsingular. ${ }^{9}$

Because the matrix $\boldsymbol{A}$ is nonsymmetric, some of its eigenvalues can be complex, but with pairs of complex conjugate values, when the fission source terms make it more nonsymmetric and less diagonally dominant. For nonmultiplicative media all eigenvalues are real and positive.

The eigenvalues and eigenvectors (right and left) of the multigroup matrix $\boldsymbol{A}$, which can be computed by the double precision real or complex subroutines DGEEV and ZGEEV of the LAPACK library, ${ }^{11}$ as well as the $\boldsymbol{R}$ and $\boldsymbol{R}^{-1}$ diagonalizing matrices, verify

$$
\begin{aligned}
\boldsymbol{A}\left|\boldsymbol{u}_{m}\right\rangle & =\lambda_{m}\left|\boldsymbol{u}_{m}\right\rangle, \\
\left\langle\boldsymbol{v}_{m}\right| \boldsymbol{A} & =\left\langle\boldsymbol{v}_{m}\right| \lambda_{m}, \\
\boldsymbol{R}^{-1} & =\left[\boldsymbol{u}_{m}\right], \\
\boldsymbol{R} & =\left[\boldsymbol{v}_{m}\right]^{\boldsymbol{T}^{*},},
\end{aligned}
$$

and

$$
\boldsymbol{R A} \boldsymbol{R}^{-1}=\left[\lambda_{m}\right]_{\mathrm{diag}} .
$$

The number of eigenmodes $m$ is the number of groups $G$, and some of them can be complex by pairs of complex conjugate values and vectors. In this case the real (double) DGEEV subroutine returns the real part in column $m$ and the imaginary part in column $m+1$. The matrix of left eigenvectors should be transpose-conjugated and renormalized to be the inverse of the matrix of right eigenvectors.

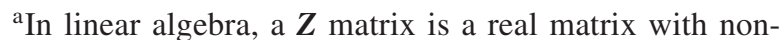
positive off-diagonal entries. A $\boldsymbol{Z}$ matrix is called an $\boldsymbol{M}$ matrix if the real part of any eigenvalue is nonnegative ( $\boldsymbol{M}$ matrices have many other equivalent definitions; they form a subclass of $\boldsymbol{Z}$ matrices that have important implications in the iterative method convergence).
Then, the modal and the physical group fluxes and sources are transformed by

$$
\begin{aligned}
\left|\boldsymbol{\psi}_{m}\right\rangle & =\boldsymbol{R}\left|\boldsymbol{\phi}_{g}\right\rangle, \\
\left|\boldsymbol{\phi}_{g}\right\rangle & =\boldsymbol{R}^{-1}\left|\boldsymbol{\psi}_{m}\right\rangle, \\
\left|\boldsymbol{s}_{m}\right\rangle & =\boldsymbol{R} \boldsymbol{D}^{-1}\left|\boldsymbol{S}_{g}\right\rangle,
\end{aligned}
$$

and

$$
\left|\boldsymbol{S}_{g}\right\rangle=\boldsymbol{D} \boldsymbol{R}^{-1}\left|\boldsymbol{s}_{m}\right\rangle
$$

And, the system of $G$ coupled multigroup diffusion equation (1) is reduced to the $G$ uncoupled modal equations:

$$
\begin{gathered}
\nabla^{2} \psi_{m}(\boldsymbol{r})-\lambda_{m} \psi_{m}(\boldsymbol{r})=-\boldsymbol{s}_{m}(\boldsymbol{r}) ; \quad m=1, G . \\
\text { II.B. Solution of the Modal Equations in } \\
\text { One-Dimensional Plane Geometry, } \\
\text { No Sources: Chao's Relations }
\end{gathered}
$$

In 1-D plane geometry, modal equations (4) are ordinary second-order differential equations, with exponential solutions of exponent $\alpha_{m}$, with $\alpha_{m}^{2}=\lambda_{m}$. If the eigenvalues $\lambda_{m}$ and $\lambda_{m+1}$ are complex conjugate, so are the exponents $\alpha_{m}$ and $\alpha_{m+1}$. The general solution of the modal homogeneous equations in this geometry is (with complex constants $A_{m}$ and $B_{m}$ )

$$
\begin{aligned}
\psi_{m}(x) & =A_{m} e^{+\alpha_{m} x}+B_{m} e^{-\alpha_{m} x}, \\
\alpha_{m+1} & =\alpha_{m}^{*},
\end{aligned}
$$

and

$$
\psi_{m+1}(x)=\psi_{m}^{*}(x)
$$

If the complex exponent is $\alpha_{m}=a+b i$, then the general solution of the complex modal flux $\psi_{m}$ can be expressed, in terms of four real constants and functions, as

$$
\begin{aligned}
\psi_{m}(x)= & c_{m} \cosh a x \cos b x+d_{m} \sinh a x \cos b x \\
+ & c_{m+1} \cosh a x \sin b x+d_{m+1} \sinh a x \sin b x \\
+ & i\left(-d_{m+1} \cosh a x \cos b x\right. \\
& \quad-c_{m+1} \sinh a x \cos b x+d_{m} \cosh a x \sin b x \\
& \left.+c_{m} \sinh a x \sin b x\right) .
\end{aligned}
$$

The Chao relations ${ }^{1}$ are obtained by calculating, now in full complex algebra, the modal flux and net current at the node interfaces $(x=[0, H]$ or $[-H / 2, H / 2])$ and then solving for the complex constants $A_{m}$ and $B_{m}$ and substituting in the node-average flux 


$$
\psi_{m}(0)=A_{m}+B_{m}
$$

and

$$
\begin{gathered}
2 A_{m}=\psi_{m}(0)-J_{m}(0) / D \alpha_{m} \\
J_{m}(0)=-D \alpha_{m}\left(A_{m}-B_{m}\right)
\end{gathered}
$$

and

$$
\begin{aligned}
2 B_{m}= & \psi_{m}(0)+J_{m}(0) / D \alpha_{m} \\
\alpha_{m} H \bar{\psi}_{m}= & A_{m}\left(e^{+\alpha_{m} H}-1\right)-B_{m}\left(e^{-\alpha_{m} H}-1\right) \\
= & \psi_{m}(0) \frac{e^{+\alpha_{m} H}-e^{-\alpha_{m} H}}{2} \\
& -\frac{J_{m}(0)}{D \alpha_{m}}\left(\frac{e^{+\alpha_{m} H}+e^{-\alpha_{m} H}}{2}-1\right) .
\end{aligned}
$$

Reordering, we obtain the generalized Chao's relation for complex eigenvalues:

$$
\psi_{m}\left(\mp \frac{H}{2}\right)=C_{m}^{f} \bar{\psi}_{m} \pm C_{m}^{j} \frac{H}{2} D^{-1} J_{m}\left(\mp \frac{H}{2}\right),
$$

where the scalar modal coefficients $C_{m}^{f}$ and $C_{m}^{j}$, real or complex, are given by

$$
C_{m}^{f}=\frac{2 \alpha_{m} H}{e^{+\alpha_{m} H}-e^{-\alpha_{m} H}}
$$

and

$$
C_{m}^{j}=\frac{e^{+\alpha_{m} H}+e^{-\alpha_{m} H}-2}{e^{+\alpha_{m} H}-e^{-\alpha_{m} H}} \frac{2}{\alpha_{m} H} .
$$

Finally, transforming from the modal fluxes to the physical group fluxes, using Eqs. (3), we obtain

$$
\begin{aligned}
\left|\boldsymbol{\phi}_{g}\left(\mp \frac{H}{2}\right)\right\rangle & =\boldsymbol{A}^{f}\left|\overline{\boldsymbol{\phi}}_{g}\right\rangle \pm \frac{H}{2} \boldsymbol{A}^{j} \boldsymbol{D}^{-1}\left|\boldsymbol{J}_{g}\left(\mp \frac{H}{2}\right)\right\rangle, \\
\boldsymbol{A}^{f} & =\boldsymbol{R}^{-1} \boldsymbol{C}^{f} \boldsymbol{R},
\end{aligned}
$$

and

$$
\boldsymbol{A}^{j}=\boldsymbol{R}^{-1} \boldsymbol{C}^{j} \boldsymbol{R},
$$

where the terms $\boldsymbol{C}^{f}$ and $\boldsymbol{C}^{j}$ are now the diagonal matrices of the modal coefficients (11), complex in general, resulting in full and nonsymmetric, but real, matrices $\boldsymbol{A}^{f}$ and $\boldsymbol{A}^{j}$. Formally, the first equation in Eqs. (12) is equal to the corresponding one in Chao, ${ }^{1}$ but the two defining equations in Eqs. (12) are now complex matrix products. When $H \rightarrow 0$ (fine mesh), the scalar modal coefficients from Eqs. (11) $C_{m}^{f} \rightarrow 1$ and $C_{m}^{j} \rightarrow 1$, and then from Eqs. (12) the matrices $\boldsymbol{A}^{f} \rightarrow I$ and $\boldsymbol{A}^{j} \rightarrow I$.

We have developed a procedure to deal only with real (double) matrix algebra, where the diagonalizing matrices $\boldsymbol{R}$ and $\boldsymbol{R}^{-1}$ are constructed as real, with a simple modification of the right and left eigenvector matrices returned by the SGEEV routine of LAPACK; the $\boldsymbol{C}^{f}$ and $\boldsymbol{C}^{j}$ are constructed with diagonal terms for the real modes and $2 \times 2$ nondiagonal boxes for the complex modes, thus reducing by a factor of $\sim 4$ all the matrix computations.

From Eqs. (12), the (column) vector of multigroup net currents at the interfaces is obtained, following Chao, ${ }^{1,15}$ as a function of the vectors of interface and node-average fluxes by

$$
\left|\boldsymbol{J}_{g}\left(\mp \frac{H}{2}\right)\right\rangle=\mp \boldsymbol{D A}^{j^{-1}} \frac{\boldsymbol{A}^{f}\left|\overline{\boldsymbol{\phi}}_{g}\right\rangle-\left|\boldsymbol{\phi}_{g}\left(\mp \frac{H}{2}\right)\right\rangle}{H / 2} .
$$

Equation (13) is the half-node ACMFD formula for each left and right interface. It is "exact" in the 1-D multigroup diffusion approximation assumed above. It is much like the linear finite difference discretization of the diffusion approximation (Fick's law) but extended to include the coarse-mesh and spectral effects of the analytic intranodal flux variation in space and spectra. The matrix $\boldsymbol{A}^{f}$ corrects the vector node-average fluxes in the "gradient" term of the diffusion Fick's law, and the matrix $\boldsymbol{A}^{j-1}$ corrects the diffusion coefficient diagonal matrix $D$.

But, the most remarkable property of the half-node ACMFD formula (13) in nodal multigroup diffusion, as pointed out by Chao, ${ }^{1,15}$ is that it is a full matrix-vector equation: The net currents of each group depend on the corrected diffusion coefficients multiplied (as matrixvector products) by the corrected gradients of the fluxes in all groups. The general ACMFD Fick's law, Eq. (13), is not anymore just a corrected or nonlinear synthetic scalar relation within each group, as, for example, in Ref. 14 or in other previous works reviewed in Ref. 15, but matrix-vector products coupling all groups at the node interfaces. Only if $H \rightarrow 0$ (fine mesh)-since then the matrices $\boldsymbol{A}^{f}$ and $\boldsymbol{A}^{j}$ become the identity matrix — do we recover the original (scalar) Fick's law.

\section{II.C. The ACMFD Method: Net Currents at the Interfaces of Different Homogeneous Nodes}

After considering above a single homogeneous node, we now consider the interface in one dimension between two different homogeneous nodes, denoted by the subindexes $n$ and $n+1$, and apply the continuity condition of the physical fluxes, given by Eqs. (12), at the common right interface of node $n$ and the left interface of node $n+1$ : 


$$
\begin{aligned}
\left|\boldsymbol{\phi}_{n}\left(\frac{H}{2}\right)\right\rangle= & \boldsymbol{A}_{n}^{f}\left|\overline{\boldsymbol{\phi}}_{n}\right\rangle-\frac{H}{2} \boldsymbol{A}_{n}^{j} \boldsymbol{D}_{n}^{-1}\left|\boldsymbol{J}_{n}\left(\frac{H}{2}\right)\right\rangle \\
= & \left|\boldsymbol{\phi}_{n+1}\left(-\frac{H}{2}\right)\right\rangle=\boldsymbol{A}_{n+1}^{f}\left|\overline{\boldsymbol{\phi}}_{n+1}\right\rangle \\
& +\frac{H}{2} \boldsymbol{A}_{n+1}^{j} \boldsymbol{D}_{n+1}^{-1}\left|\boldsymbol{J}_{n+1}\left(-\frac{H}{2}\right)\right\rangle .
\end{aligned}
$$

Applying as well the continuity condition to the net currents at the common interface, the last are directly obtained from Eq. (14) as

$$
\begin{aligned}
\left|\boldsymbol{J}_{n+1 / 2}\right\rangle= & \left(\boldsymbol{A}_{n}^{j} \boldsymbol{D}_{n}^{-1}+\boldsymbol{A}_{n+1}^{j} \boldsymbol{D}_{n+1}^{-1}\right)^{-1} \\
& \times \frac{\boldsymbol{A}_{n}^{f}\left|\overline{\boldsymbol{\phi}}_{n}\right\rangle-\boldsymbol{A}_{n+1}^{f}\left|\overline{\boldsymbol{\phi}}_{n+1}\right\rangle}{H / 2} .
\end{aligned}
$$

Equation (15) substituted in Eq. (1) integrated over each node of the system constitutes the highest-order and simplest discretization of the multigroup diffusion equations provided by the ACMFD method. It is strictly exact for 1-D multigroup diffusion problems with homogeneous regions or nodes, and we will show later how it can keep quite high accuracy for heterogeneous nodes and 3-D problems. It is the simplest because it reduces to a minimum the number of unknowns, the average fluxes per node and group, and the final resulting linear matrix to solve is block-diagonal, with blocks of $G \times G$ nonzero elements and $(1+$ nfaces $)$ nonzero block-diagonals, where nfaces is the number of faces per node.

Equation (15) gives the vector of group net currents at each node interface in terms of the difference of two matrix-vector products of two different corrected diffusion matrices by the vector of group fluxes averaged on the two nodes at each side of the interface. Since the difference (15) has nonsymmetric coefficients, the matrix of the final linear system is nonsymmetric.

\section{II.D. Heterogeneous Nodes: Interface Flux Discontinuity Factors}

Although the above analysis is only rigorous for homogeneous nodes, we showed in our previous work, ${ }^{5,6}$ that by introducing the heterogeneity effects through interface-dependent scalar flux discontinuity factors, as first introduced by Koebke, ${ }^{19}$ for each node type, precalculated by single-assembly lattice calculations and a few parametrical colorsets, the ACMFD method can be effectively and accurately implemented for actual and quite heterogeneous operating reactors.

In this work, we introduce the interface flux discontinuity jumps or differences in the modal space for each node, as scalar jumps per mode in the left side of Eq. (10), which were derived for a homogenized node. The actual heterogeneous physical fluxes at the node interfaces, cal- culated by a higher-order and detailed calculation (such as multigroup transport for the detailed fuel assembly, colorset, or core-reflector problem), are first transformed to the modal space using Eqs. (3) and then related to the homogeneous modal flux in Eq. (10) by the modal discontinuity difference at each interface by

$$
\boldsymbol{R}\left|\boldsymbol{\phi}_{g}^{\text {het }}\left(\mp \frac{H}{2}\right)\right\rangle=\left|\psi_{m}^{\text {het }}\left(\mp \frac{H}{2}\right)\right\rangle
$$

and

$$
\psi_{m}^{\text {het }}\left(\mp \frac{H}{2}\right)=\psi_{m}^{\text {hom }}\left(\mp \frac{H}{2}\right)+d_{m}\left(\mp \frac{H}{2}\right) .
$$

Then, at each interface $( \pm H / 2$ above, to be dropped in the following), the heterogeneous (continuous) physical group fluxes are related to the homogeneous (discontinuous) analytical group fluxes again by a matrix-vector product that couples all groups:

$$
\left|\boldsymbol{\phi}_{g}^{\text {het }}\left(\mp \frac{H}{2}\right)\right\rangle=\left|\boldsymbol{\phi}_{g}^{\text {hom }}\left(\mp \frac{H}{2}\right)\right\rangle+\left|\boldsymbol{F}_{d}\right\rangle
$$

and

$$
\left|\boldsymbol{F}_{d}\right\rangle=\boldsymbol{R}^{-1}\left|\boldsymbol{d}_{m}\right\rangle
$$

where

$$
\begin{aligned}
\left|\boldsymbol{d}_{m}\right\rangle= & \text { vector of modal discontinuity jumps at the } \\
& \text { interface, defined by Eqs. (16), which can } \\
& \text { be real or complex, since the modal fluxes } \\
& \text { are complex for the modes with complex } \\
& \text { eigenvalues } \\
\left|\boldsymbol{F}_{d}\right\rangle= & \text { new (real) vector of physical flux disconti- } \\
& \text { nuity jumps at each node interface. }
\end{aligned}
$$

In Eq. (14), the continuity at the interface between nodes $n$ and $n+1$ should be now applied to the heterogeneous interface physical group fluxes, so that the terms for each node appear now decreased by the vector $\boldsymbol{F}_{d}$ of the respective node and interface. Putting together Eqs. (10), (12), (16), and (17), the previous nodal coupling equation (15) is corrected to include as well the differences of the discontinuity vectors $\boldsymbol{F}_{d}$ at both sides of the nodal interface. We are evaluating the merits of this formulation as compared with our previous one, which used scalar physical flux discontinuity factors.

\section{THE ACMFD METHOD FOR MULTIDIMENSIONAL PROBLEMS}

The ACMFD method can be extended for 2-D (and $3-D)$ problems, in rectangular $X-Y[-Z]$ or triangular $[-Z]$ geometries, using two alternative schemes: the transverse flux integration and the incomplete analytic flux 
expansion. We will show their equivalence, and subtle differences, as introduced by Chao. ${ }^{1,2}$ For simplicity, only the 2-D rectangular $X-Y$ case will be considered below because the extension to $3-\mathrm{D}$ rectangular and triangular $Z$ geometry follows with the same guidelines and are under implementation and testing.

\section{III.A. The Transverse Flux Integration Method: Approximations of Transverse Leakage}

The transverse flux integration method was introduced in Ref. 20 for the NEM, with its nonlinear extension, ${ }^{21}$ and by Chao in the ACMFD method. ${ }^{1}$ Its analytical derivation is straightforward for rectangular $X-Y-Z$ geometries because the volumes of the transverse flux integrals are constant, and then, the transverse leakage terms are just the difference of the net currents at the transverse interfaces divided by this constant volume. The 2-D or 3-D neutron diffusion equation (1), within any homogenized region or node, is then formally transverse integrated (i.e., in $y$ and $z$ ) over the node and averaged over the transverse volumes to yield the reduced 1-D flux equations, as Eq. (1) with $\boldsymbol{r}=x$, or the separated 1-D modal equations, as Eqs. (4) also with $r=x$. But, now, in the right side of Eqs. (1) and (4), the physical independent neutron source $S_{g}(x)$ also includes the transverse leakage $L_{g}(x)$, both per unit volume and group, and the modal independent term is just given by the matrix relations (3) as

$\left|s_{m}(x)\right\rangle=\boldsymbol{R} \boldsymbol{D}^{-1}\left|S_{g}^{*}(x)\right\rangle$

and

$$
\begin{aligned}
S_{g}^{*}(x)=S_{g}(x)+[ & J_{y}^{-H_{y} / 2}(x)-J_{y}^{+H_{y} / 2}(x)+J_{z}^{-H_{z} / 2}(x) \\
& \left.-J_{z}^{+H_{z} / 2}(x)\right] / H_{y} H_{z} .
\end{aligned}
$$

The solution of the modal equations (4) with this right side in 1-D plane geometry is now given by the general solution (5) of the homogeneous equation plus a particular solution $p(x)$ of Eqs. (4) with Eq. (18):

$$
\psi_{m}(x)=A_{m} e^{+\alpha_{m} x}+B_{m} e^{-\alpha_{m} x}+p_{m}(x)
$$

and

$$
\frac{d^{2}}{d x^{2}} p_{m}(x)-\lambda_{m} p_{m}(x)=-\boldsymbol{s}_{m}(x) .
$$

Note that for complex (conjugate) eigenvalues, the above modal relations also are complex and conjugate for modes $m$ and $m+1$. Eliminating the constants, as in Eqs. (7), (8), and (9), the modal Chao's relation (10) with the particular solution term become, in general complex,

$$
\begin{aligned}
\psi_{m}(\mp & \left.\frac{H}{2}\right)-p_{m}\left(\mp \frac{H}{2}\right) \\
= & C_{m}^{f}\left[\bar{\psi}_{m}-\bar{p}_{m}\right] \\
& \pm C_{m}^{j} \frac{H}{2}\left[D^{-1} J_{m}\left(\mp \frac{H}{2}\right)+p_{m}^{\prime}\left(\mp \frac{H}{2}\right)\right],
\end{aligned}
$$

where definitions (11) hold and the interface values, node averages, and derivatives (with $x$ ) of the modal particular solutions $p_{m}(x)$ just subtract to the respective values of the modal fluxes. By using relations (3) and (18), Eq. (20) is transformed into matrix-vector product relations of the physical group fluxes, transverse currents, and independent source (if present), similar to Eqs. (12) through (15).

In 2-D and 3-D problems, a nonlinear iterative scheme can be implemented as in NEM: The transverse leakage terms are interpolated from the previous nodal solution, using a quadratic or cubic fit of the transverse net currents along the lines or planes of node interfaces. From Eq. (20) we see how this fit should be implemented for best achievable accuracy: using the node interface averaged transverse net currents and their values and derivatives at each node corner or aristae. Following our numerical experience, ${ }^{5,6}$ this nonlinear scheme converges quite accurately and quickly, at the rate of the criticality searches and the nonlinear and thermalhydraulic feedbacks in realistic large and heterogeneous cores.

\section{III.B. The Incomplete Analytic Flux Expansion Method}

Another alternative $\mathrm{e}^{1,2,16-18}$ for multidimensional problems is the incomplete analytic flux expansion method applied to the modal equations (4), which have the form of the wave equation in quantum mechanics (Schrödinger equation for free particles in three dimensions). The general solution of the homogeneous modal equations (4) is given by any linear combination of the plane waves, Eqs. (5), but now in any direction of the real and complex 3-D planes:

$$
\psi_{m}(\boldsymbol{r})=\int_{4 \pi} A_{m}\left(\boldsymbol{k}_{m}\right) e^{\boldsymbol{k}_{m} \boldsymbol{r}} d \boldsymbol{k}_{m}
$$

and

$$
\left\|\boldsymbol{k}_{m}\right\|^{2}=\gamma_{m} .
$$

The vector $\boldsymbol{k}_{m}$ is now, in general, a complex in the 2-D or 3 -D space, with the condition that its norm is the corresponding eigenvalue $\gamma_{m}$ of the multigroup matrix, which can be real negative, null, or positive for the fundamental mode and real positive or complex of the positive real part for the other modes, as established in Sec. II.A. 
In Cartesian coordinates the scalar product can be written as $\boldsymbol{k r}=k_{x} x+k_{y} y+k_{z} z$, with $k_{x}^{2}+k_{y}^{2}+k_{z}^{2}=$ $k^{2}=\gamma_{m}$, where all terms are in general complex, even if $\gamma_{m}$ is real. For practical application the complete, infinite, modal flux expansion (21) has to be truncated to an incomplete, but finite, number of terms. The lowestorder approximation is obtained when only the plane waves propagating in both directions along the $X$ or $Y$ or $Z$ axis are considered:

$$
\begin{aligned}
\psi(x, y, z)= & A_{1} e^{k x}+A_{2} e^{-k x}+A_{3} e^{k y}+A_{4} e^{-k y} \\
& +A_{5} e^{k z}+A_{6} e^{-k z} .
\end{aligned}
$$

To obtain the ACMFD modal relation for a given node and face, we shall determine the six constants by imposing the six following conditions for the face average modal flux at that face and the face-averaged transverse net currents at five of the six node faces, excluding the opposite face to the one considered. Integrating Eq. (22) over the node and substituting the six constants, we obtain a similar ACMFD modal relation (20) with constant transverse leakage (flat interface currents).

The next order in the plane wave expansion is to consider, in addition to the previous waves, the plane waves propagating in both directions along the two diagonal lines across the node center and its four corners in two dimensions, where $k_{x}=k_{y}=k / \sqrt{2}$, giving the four next additional terms to Eq. (22):

$$
\begin{aligned}
\psi(x, y)= & A_{1} e^{k x}+\cdots+A_{4} e^{-k y}+A_{5} e^{k(x+y) / \sqrt{2}} \\
& +A_{6} e^{k(x-y) / \sqrt{2}}+A_{7} e^{-k(x+y) / \sqrt{2}} \\
& +A_{8} e^{-k(x-y) / \sqrt{2}} .
\end{aligned}
$$

This is the order of expansion used in the AFEN method. ${ }^{16-18}$ In this case we can determine the additional four constants by several choices of constraints obtained from the nodal flux solution of the previous iteration. Our choice, which consistently with the transverse integration shown above has the potential for an accurate scheme, is to impose the conditions of the net transverse currents and their derivatives at the two corners of the interface being considered. Thus, if we consider the interface at $x=H / 2, y \in[-H / 2,+H / 2]$, we impose the following eight conditions: the modal average flux and net current $J_{x}$ at $x=H / 2$, the modal average net currents $J_{y}$ at $y= \pm H / 2$, and the modal point net currents $J_{y}$ and their derivatives with $x$ at the corners $(x=H / 2$, $y= \pm H / 2$ ). This yields, after a quite elaborated algebra, a similar result to the one obtained in Eq. (20). Since this scheme has more local information around each interface, it provides, in principle, an accurate and fast converging nonlinear scheme. The extension to 3-D rectangular and triangular $Z$ geometries is being implemented along the same guidelines.

\section{NUMERICAL AND GRAPHICAL ANALYSIS OF THE MULTIGROUP EIGENPROBLEM}

We have implemented and tested the above scheme for multigroup diffusion constants sets generated in 2, 3, 4, 8, 12, and 24 groups for realistic PWR fuel assemblies and reflectors. First, we present the numerical and graphical results for the multigroup eigenvalue problem in these homogenized regions. Numerical validation 1-D and 2-D results shall be provided later.

For the multigroup cross-section generation, we considered a model core-reflector problem:

1. inner core of midburnup PWR fuel: from 0 to $131.05 \mathrm{~cm}$

2. outer core of high-burnup PWR fuel: in two nodes of $10.803 \mathrm{~cm}$ width

3. core shroud plate of Type 304 stainless steel: $2.233 \mathrm{~cm}$ thickness

4. borated water reflector: up to $3 \times 10.803 \mathrm{~cm}$ of core-steel boundary.

We calculated this model problem in 1-D cylindrical geometry with the WIMS-D code ${ }^{22}$ using $S_{6}$ in 24 neutron transport groups and realistic material conditions and dimensions for a PWR at nominal hot full power [4.65 wt $\% 17 \times 17$ fuel assemblies, at 22.9 and 43.1 $\mathrm{GWd} / \mathrm{t}$ burnup, $307^{\circ} \mathrm{C}$ core inlet water temperature, $2170 \mathrm{ppm}$ boron and 2940.6-MW(thermal) core power]. The numbers of fuel rods used in the one inner and two outer core regions were 30546,5244 , and 5658, respectively, for the WIMS cluster model (array and rodsub options).

The diffusion coefficients and cross sections, as well as the region-integrated fluxes, in 24 groups were extracted from the WIMS calculations for three homogeneous regions of the model problem: outer fuel, steel, and water reflectors. The 24-group fluxes were used to collapse the cross sections to different numbers of groups: $24,12,8,6,4,3$, and 2 , where we compute the multigroup diffusion matrices, eigenvalues, and eigenvectors of the analytic multigroup diffusion theory, as well as Chao's and other response matrices for 1-D plane geometry.

\section{IV.A. Analysis of the Multigroup Matrix: Trace, Norm, and Spectral Radius}

First, we analyze the eigenvalue spectra of the multigroup diffusion matrices of the above three materials for several numbers of groups. The maximum information that can be directly obtained from the multigroup matrices is given by the spectral theorems of linear algebra. ${ }^{9,10}$ The simplest bound is that the absolute value of any eigenvalue should be less than or equal to any matrix norm. In particular, the spectral radius, defined by the maximum absolute eigenvalue, is bounded by the 


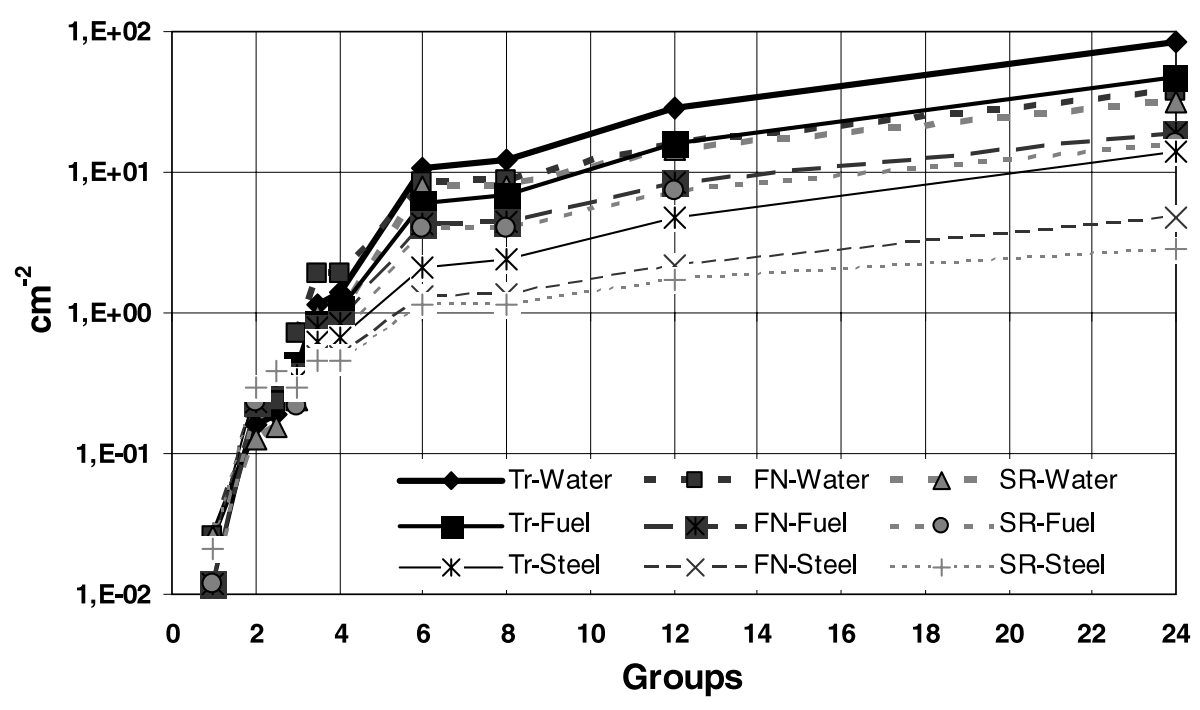

Fig. 1. Trace, F-norm, and spectral radius.

Fröbenius norm. Figure 1 plots the traces, F-norms, and spectral radius for the multigroup matrices of the three materials given above with several numbers of groups, where a trend of increasing the above bounds with the number of groups is clearly observed. But, a more precise localization of all the eigenvalues can be obtained from the following theorem.

\section{IV.B. Analysis of the Multigroup Matrix Spectrum: Gerschgorin Disks and Eigenvalues}

The Gerschgorin theorem ${ }^{9,10}$ says that any eigenvalue of any matrix $\boldsymbol{A}$ is located inside one of the closed circles or disks of the complex plane centered at the diagonal elements $a_{i i}$ (in the real positive axis for real $Z$ or $\boldsymbol{M}$ matrices) and their radii are the sums of the absolute values of all the nondiagonal elements in the same row or column. In other words, the union of the Gerschgorin disks contains the spectrum of all the eigenvalues $\sigma(A)$. A corollary is that if there are $m$ disks whose union $S$ is disjoint from all other disks, then $S$ contains exactly $m$ eigenvalues. If all disks were disjoint, each disk must contain exactly one eigenvalue.

Figure 2 plots the Gerschgorin disks and the eigenvalues for the multigroup diffusion matrices of the above materials (fuel, steel, and water) for the cases of 6 and 12 groups. We see that the disks for water are about twice as large as the disks for fuel, while the disks for steel are about or less that one-tenth of the water disks. The disks for 12 groups are about twice the disks for 6 groups. Only for steel is it inferred that the matrix in nonsingular.

The differences in the spatial and spectral responses within each material region are given by the $\alpha_{m}$ eigenvalues $\left(\alpha_{m}^{2}=\lambda_{m}\right)$ and the $\boldsymbol{R}$ and $\boldsymbol{R}^{-1}$ matrices of eigenvectors. In Figure 3, we plot the real part of the $\alpha_{m}$ for the different modes calculated for the fuel, water, and steel materials of the model problem. We see in Fig. 3 how the first mode $\alpha_{m}$ eigenvalue (fundamental mode) is quite different for these materials (positive and large for water to near zero for fuel). The next eigenvalues always have a positive and increasing real part, which are not so different among the materials. The $\alpha_{m}$ modes from second to sixth or so increase almost linearly in this logarithmic plot, showing how the associated modal terms vanish much faster with the distance from the boundaries.

\section{ANALYTIC MULTIGROUP DIFFUSION AT THE CORE-REFLECTOR BOUNDARY AND OUTER REFLECTORS}

A strong spectral change is observed at the corereflector boundaries, which intensifies the effects of the reflector heterogeneities, such as the presence of a thick steel plate as a radial core support or shroud. Similar or more complex heterogeneities are found in both the radial and axial reflectors at specific elevations or reactor designs.

Figure 4 shows the 24-group transport fluxes inside regions 2,3 , and 4 of the model problem defined above, from 131.05 to $185.06 \mathrm{~cm}$. We see here the fundamental mode exponential flux decay, well inside the homogeneous regions (outer subcritical core and water reflector), and the strong transient modes at the core-steel-water boundaries, with a quite different behavior in the fast and thermal groups.

Figure 5 shows the normalized flux spectra per unit energy in 24 groups at selected region interfaces. The change of the neutron spectrum from the core to the water reflector is quite large and rather noticeable at the steel-water interface. 
Gerschgorin Circles and Eigenvalues in 6 groups
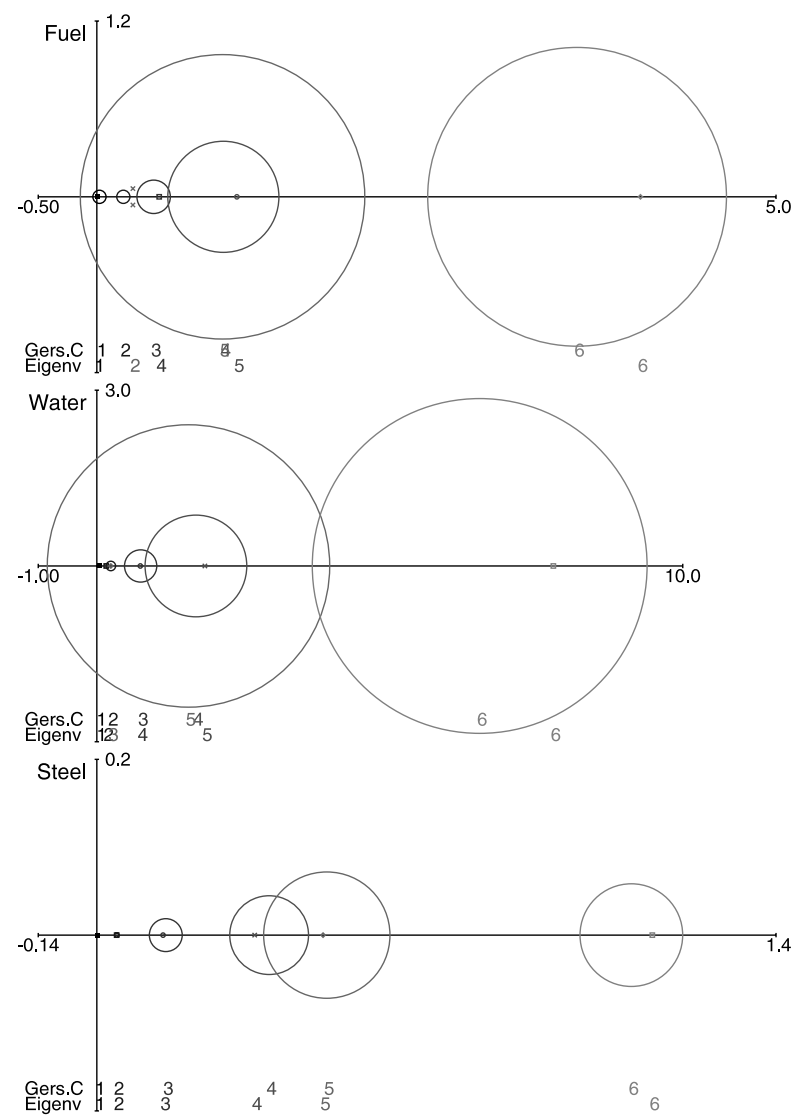

Gerschgorin Circles and Eigenvalues in 12 groups
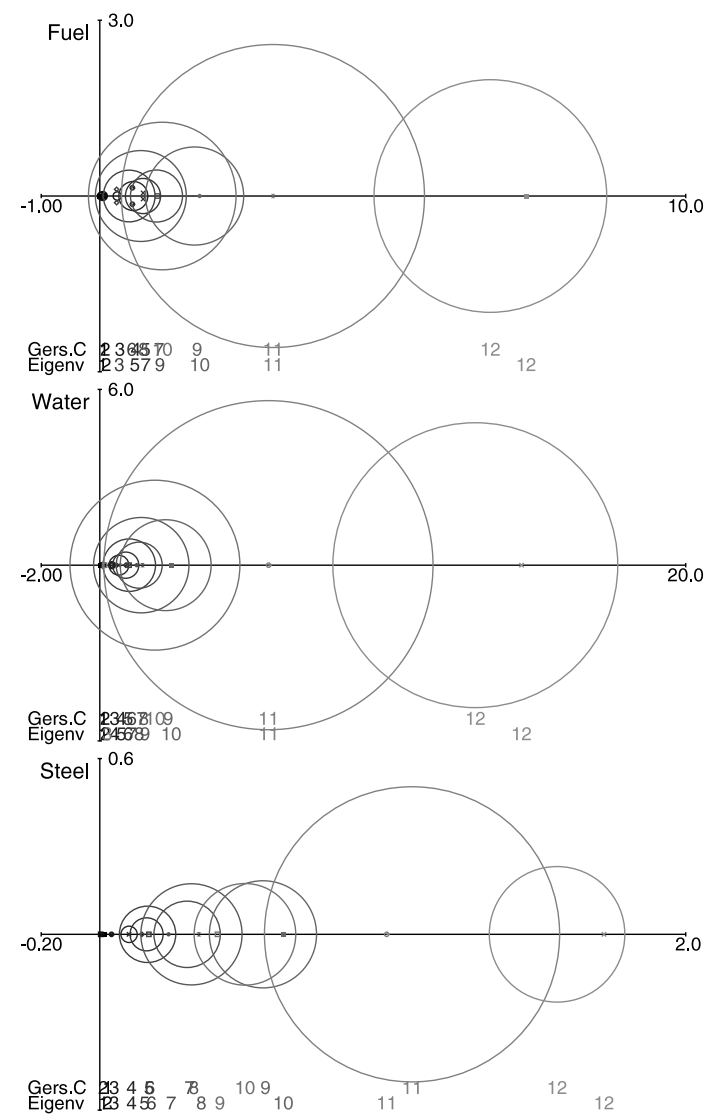

Fig. 2. Gerschgorin disks and eigenvalues of multigroup diffusion matrices in 6 and 12 groups for fuel, water, and steel.

If the reflector is explicitly accounted for in the nodal core calculations, adequate "equivalent" homogenized cross sections and discontinuity factors are required. This requires costly variational fitting methods or linear and

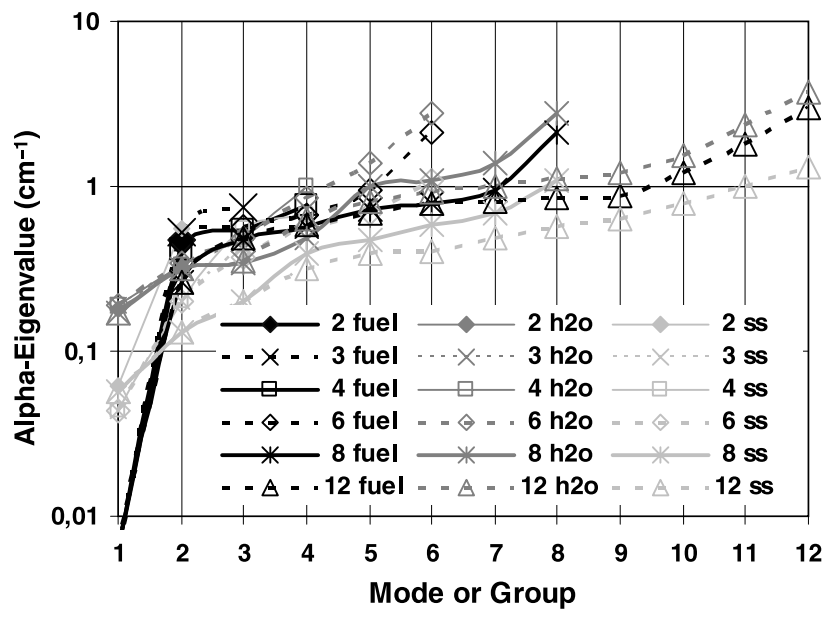

Fig. 3. The $\alpha_{m}$ eigenvalues versus eigenmodes. scalar approximations that are not accurate for all the design or operating conditions.

Through the introduction of appropriate core-reflector boundary conditions, it is not necessary to explicitly account for the reflector in the nodal core calculations. Thus, the numerical effort of solving the diffusion equation can be concentrated in the domain of interest, reducing computational requirements. Multigroup albedos can be used to define those boundary conditions, but albedos are scalars that depend on the core properties and must be generated for each specific core loading. If the reflector is taken into account by multigroup response matrices, which relate the vectors of surface fluxes and net currents at the core-reflector boundary, they should couple properly all groups.

A reflector response matrix method that conserves the heterogeneous response at core-reflector interfaces in nodal calculations is introduced here. The response matrices $[\beta]$ relate the vectors of net currents and surface fluxes at each fuel-reflector interface, coupling properly all groups:

$$
\left.\left.\mid \boldsymbol{\phi}_{g}(\text { interface })\right\rangle=[\beta] \mid \boldsymbol{J}_{g}(\text { interface })\right\rangle \text {. }
$$




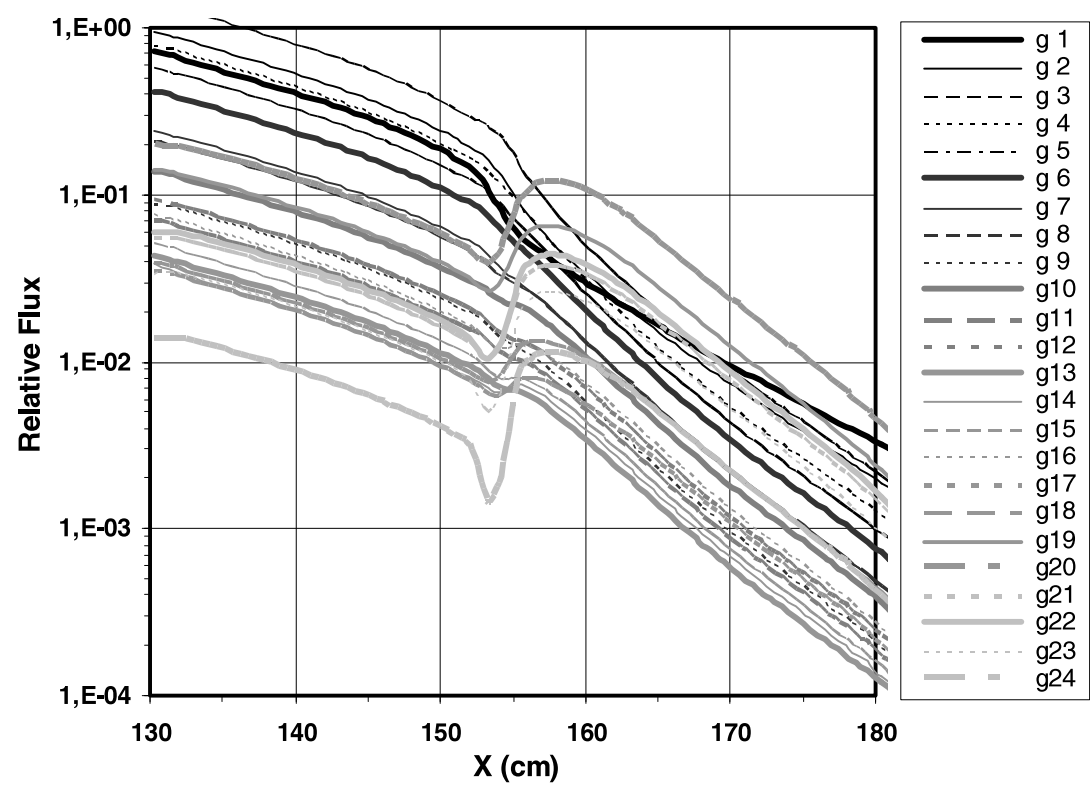

Fig. 4. The 24-group fluxes at PWR core reflector.

Plugging Eq. (24) into Eq. (14), we obtain the boundary conditions that relate the net currents and the average fluxes in the last active core region to be used in the ACMFD reactor calculations. Proceeding this way, the problem lies in how to calculate the response matrix at each fuel-reflector interface, with realistic 1-D and 2-D (corners) effects in heterogeneous reflectors.

\section{V.A. One-Dimensional Slab Infinite Reflector Case}

The outer water reflector is so thick that it behaves as an infinite reflector, where the positive real exponential terms in Eqs. (4) should vanish, reducing to

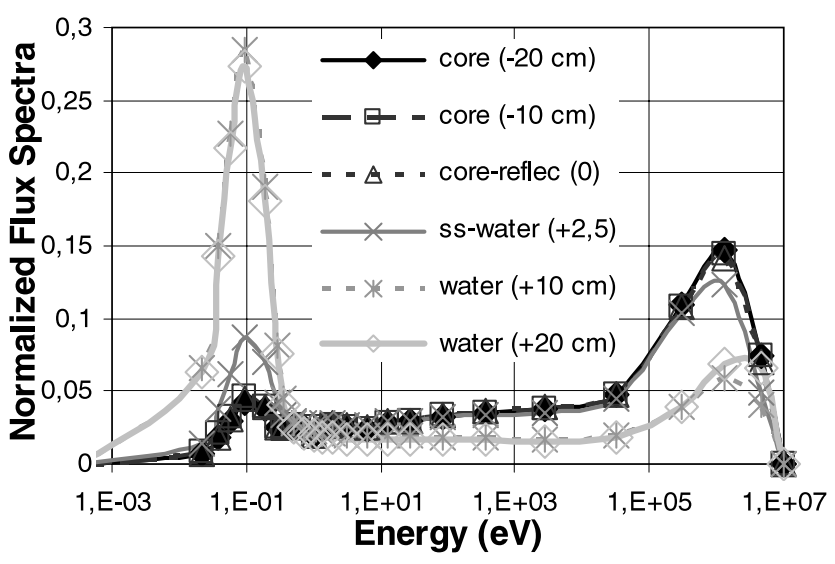

Fig. 5. Flux spectra in 24 groups at core reflector and outer reflector.

$$
\psi_{m}(x)=B_{m} e^{-\alpha_{m} x}
$$

and

$$
\alpha_{m}=\sqrt{\lambda_{m}} \quad\left(\alpha_{m} \text { real } \Leftarrow \lambda_{m} \text { positive }\right) .
$$

The modal scalar net currents are now

$$
J_{m}(x)=+D \alpha_{m} B_{m} e^{-\alpha_{m} x}=D \alpha_{m} \psi_{m}(x) .
$$

Then, the current-to-flux ratio is a scalar constant, in the modal space, inside the infinite reflector. To go back to the physical space, we use the relations (3) in Eq. (26), obtaining a constant matrix relation (or "response matrix") among the multigroup flux and the net current vectors:

$$
\left|\boldsymbol{\phi}_{g}(x)\right\rangle=\left[\beta_{\infty}\right]\left|\boldsymbol{J}_{g}(x)\right\rangle
$$

and

$$
\left[\boldsymbol{\beta}_{\infty}\right]=\boldsymbol{R}^{-1}\left[\alpha_{m}\right]^{-1} \boldsymbol{R} \boldsymbol{D}^{-1},
$$

where $\left[\alpha_{m}\right]$ and $\boldsymbol{D}$ are now diagonal matrices and $\left[\beta_{\infty}\right]$ is a full matrix. In this case of 1-D infinite reflector (Fig. 6), the response matrix only depends on the reflecting properties, being nondependent on the multiplicative region, that is, nondependent on the core loading pattern and burnup.

In Tables I and II, we give these reflector response matrices calculated in six groups for the infinite reflector case of the water and steel regions of the model problem. We see how these response matrices are quite different for both materials. The group coupling is much higher in 


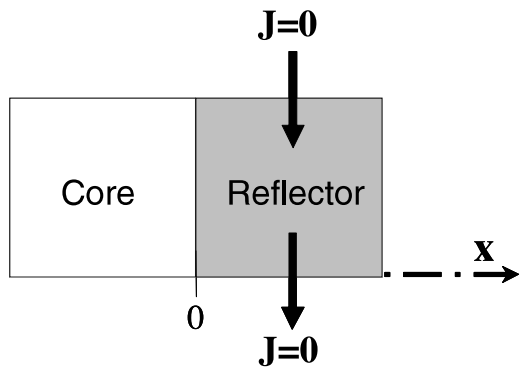

Fig. 6. One-dimensional infinite reflector geometry.

the water reflector, as the current-to-flux ratios or the albedos in the fast groups.

\section{V.B. One-Dimensional Multislab Finite plus Infinite Reflector Case}

For the case of a reflector of finite thickness $h$, as is the steel plate of our model problem, we can use Chao's relations (11) at the outer interface, together with the response matrix of the infinite water reflector, Eqs. (27), at the same interface:

$$
\begin{aligned}
\left|\boldsymbol{\phi}_{g}\left(\frac{h}{2}\right)\right\rangle & =\boldsymbol{A}^{f}\left|\overline{\boldsymbol{\phi}}_{g}\right\rangle-\frac{h}{2} \boldsymbol{A}^{j} \boldsymbol{D}_{g}^{-1}\left|\boldsymbol{J}_{g}\left(\frac{h}{2}\right)\right\rangle \\
& =\left[\beta_{\infty}\right]\left|\boldsymbol{J}_{g}\left(\frac{h}{2}\right)\right\rangle,
\end{aligned}
$$

TABLE I

Matrix $[\beta]=|\phi\rangle /|\boldsymbol{J}\rangle$ in Six Groups

\begin{tabular}{|c|c|c|c|c|c|c|}
\hline & 1 & 2 & 3 & 4 & 5 & 6 \\
\hline 1 & {$[3.051$} & 0 & 0 & 0 & 0 & 0 \\
\hline 2 & .737 & 2.614 & 0 & 0 & 0 & 0 \\
\hline 3 & .246 & .591 & 1.932 & .018 & .005 & .005 \\
\hline 4 & .233 & .466 & .744 & 2.148 & .353 & .322 \\
\hline 5 & 1.849 & 3.278 & 4.413 & 5.344 & 7.466 & 5.897 \\
\hline 6 & .671 & 1.185 & 1.586 & 1.899 & 2.257 & 4.553 \\
\hline
\end{tabular}
for Water Infinite 1-D Reflector

TABLE II

Matrix $[\beta]=|\phi\rangle /|\boldsymbol{J}\rangle$ in Six Groups

\begin{tabular}{|c|c|c|c|c|c|c|}
\hline & 1 & 2 & 3 & 4 & 5 & 6 \\
\hline 1 & {$[18.245$} & 0 & 0 & 0 & 0 & 0 \\
\hline 2 & 1.371 & 13.045 & 0 & 0 & 0 & 0 \\
\hline 3 & .124 & .794 & 7.386 & .279 & .013 & .004 \\
\hline 4 & .020 & .117 & .740 & 4.855 & .149 & .036 \\
\hline 5 & .007 & .041 & .228 & .957 & 4.522 & .768 \\
\hline 6 & .001 & .004 & .021 & .079 & .266 & 3.275 \\
\hline
\end{tabular}
for Steel Infinite 1-D Reflector which gives the right currents in terms of the region averaged fluxes, as

$$
\left|\boldsymbol{J}_{g}\left(\frac{h}{2}\right)\right\rangle=\left[\frac{h}{2} \boldsymbol{A}^{j} \boldsymbol{D}_{g}^{-1}+\beta_{\infty}\right]^{-1} \boldsymbol{A}^{f}\left|\overline{\boldsymbol{\phi}}_{g}\right\rangle .
$$

Next, we use the neutron balance multigroup diffusion equation (1) integrated over this region, which relates the differences of the left and right net currents (or net leakage) to the region integrated absorptions, plus outscatters, minus the inscatters from other groups by

$$
\left|\boldsymbol{J}_{g}\left(-\frac{h}{2}\right)\right\rangle-\left|\boldsymbol{J}_{g}\left(+\frac{h}{2}\right)\right\rangle=h \boldsymbol{D} \boldsymbol{A}\left|\overline{\boldsymbol{\phi}}_{g}\right\rangle .
$$

Plugging Eq. (29) into Eq. (30), we obtain the vector of left currents as a matrix $\boldsymbol{M}$ by the vector of region average fluxes:

$$
\left|\boldsymbol{J}_{g}\left(-\frac{h}{2}\right)\right\rangle=\boldsymbol{M}\left|\overline{\boldsymbol{\phi}}_{g}\right\rangle
$$

and

$$
\boldsymbol{M}=\left[\frac{h}{2} \boldsymbol{A}^{j} \boldsymbol{D}_{g}^{-1}+\beta_{\infty}\right]^{-1} \boldsymbol{A}^{f}+h \boldsymbol{D} \boldsymbol{A} .
$$

Finally, we use the Chao relation (28), at the left interface of this region, with Eqs. (31), to obtain

$$
\begin{aligned}
\left|\boldsymbol{\phi}_{g}\left(-\frac{h}{2}\right)\right\rangle & =\boldsymbol{A}^{f}\left|\overline{\boldsymbol{\phi}}_{g}\right\rangle+\frac{h}{2} \boldsymbol{A}^{j} \boldsymbol{D}_{g}^{-1}\left|\boldsymbol{J}_{g}\left(-\frac{h}{2}\right)\right\rangle \\
& =\left[\boldsymbol{\beta}_{0}\right]\left|\boldsymbol{J}_{g}\left(-\frac{h}{2}\right)\right\rangle,
\end{aligned}
$$

where we have introduced the response matrix $\left[\beta_{0}\right]$ at the left interface of the finite steel region (at the corereflector boundary), thus defined as

$$
\left[\beta_{0}\right]=\boldsymbol{A}^{f} \boldsymbol{M}^{-1}+\frac{h}{2} \boldsymbol{A}^{j} \boldsymbol{D}_{g}^{-1} .
$$

This response matrix can then be analytically calculated in any number of groups, allowing one to replace any heterogeneous reflector in an exact way (at least for 1-D problems), as far as the cross sections of all the reflector regions are given in the full multigroup structure. Note that the response matrix is exclusively dependent on the reflector layout and not on the specific core loading. For multiple layers Eqs. (33) and (31) can be applied recursively.

To show that a homogenized reflector cannot replicate the response of a heterogeneous (multiregion) reflector, we compare in Tables III and IV the response matrices at the core-reflector boundary in six groups calculated with the Eq. (33) with the one calculated 


\section{TABLE III}

Matrix $[\beta]=|\phi\rangle /|\boldsymbol{J}\rangle$ in Six Groups at Core Boundary for Steel and Water Heterogeneous Finite 1-D Slab Reflector

\begin{tabular}{|c|c|c|c|c|c|c|}
\hline & 1 & 2 & 3 & 4 & 5 & 6 \\
\hline 1 & {$[4.224$} & 0 & 0 & 0 & 0 & 0 \\
\hline 2 & .914 & 3.547 & 0 & 0 & 0 & 0 \\
\hline 3 & .274 & .718 & 2.419 & .026 & .006 & .006 \\
\hline 4 & .198 & .444 & .753 & 2.342 & .334 & .299 \\
\hline 5 & 1.222 & 2.438 & 3.495 & 4.453 & 6.690 & 5.063 \\
\hline 6 & .423 & .840 & 1.197 & 1.505 & 1.874 & 4.152 \\
\hline
\end{tabular}

TABLE IV

Matrix $[\beta]=|\phi\rangle /|\boldsymbol{J}\rangle$ in Six Groups at Core Boundary for Steel and Water Homogeneous Finite 1-D Slab Reflector

\begin{tabular}{|c|c|c|c|c|c|c|}
\hline & 1 & 2 & 3 & 4 & 5 & 6 \\
\hline 1 & 4.751 & 0 & 0 & 0 & 0 & 0 \\
\hline 2 & .664 & 7.494 & 0 & 0 & 0 & 0 \\
\hline 3 & .173 & .504 & 5.908 & .160 & .008 & .003 \\
\hline 4 & .092 & .186 & .576 & 4.579 & .152 & .046 \\
\hline 5 & .296 & .479 & .623 & 1.178 & 4.642 & .884 \\
\hline 6 & .059 & .094 & .112 & .148 & .312 & 3.299 \\
\hline
\end{tabular}

with Eqs. (27) for the infinite steel-water reflector homogenized with the WIMS fluxes in a $10.803-\mathrm{cm}$ node (half-assembly).

\section{V.C. Two-Dimensional and \\ Three-Dimensional Pressurized Water \\ Reactor Radial Reflector in Two Groups: \\ Corner and Transverse Leakage Effects}

The radial reflector of a PWR cannot be considered as in 1-D geometry. As shown in Fig. 7, two types of

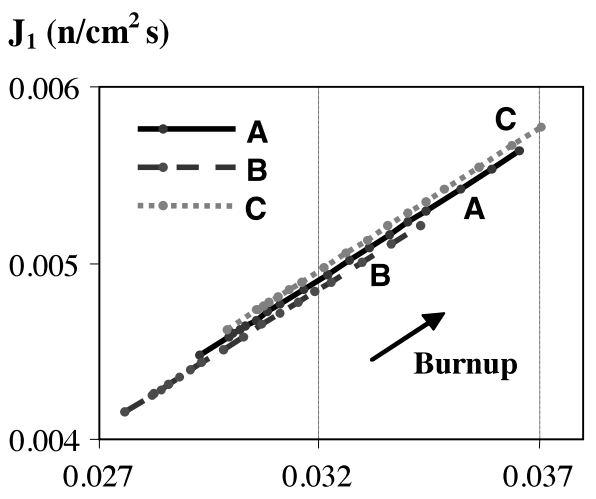

(a) $\Phi_{1}\left(\mathbf{n} / \mathrm{cm}^{2} \mathbf{s}\right)$

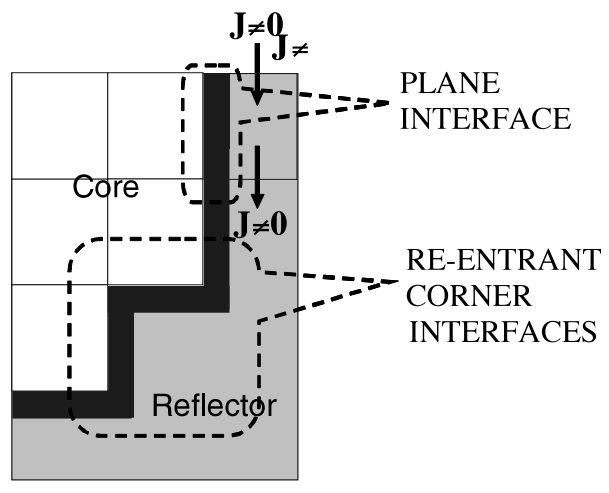

Fig. 7. Two-dimensional radial reflector geometry in PWR.

interfaces can be distinguished: plane interfaces and reentrant corner interfaces. In the former, the matrix response could be calculated through Eqs. (33) and (31) assuming 1-D geometry, even if there are nonzero transverse currents. However, in the latter, the 1-D model is not suited at all. Because of both the large mean free path and the small water reflection of fast neutrons, they can penetrate in the reflector and then reenter in the core, increasing the reflection rate. Consequently, we face a multidimensional problem.

When applying the ACMFD method in two groups to the core-reflector interfaces, the reflector response matrices were straightforward obtained in the case of 1-D geometries. In order to study the 2-D (corner and transverse leakage) core-reflector effects, detailed two-group fine-mesh diffusion and transport calculations, with explicit heterogeneous reflectors and cores, have been made.

In Fig. 8 the fast group current-to-flux ratio at the interfaces pointed out in Fig. 9 is represented along with burnup when changing the core loading. As can be seen, the responses vary with the specific core pattern, both at the plane and corner interfaces. The biggest differences

\section{$\mathrm{J}_{1}\left(\mathbf{n} / \mathrm{cm}^{2} \mathbf{s}\right)$}

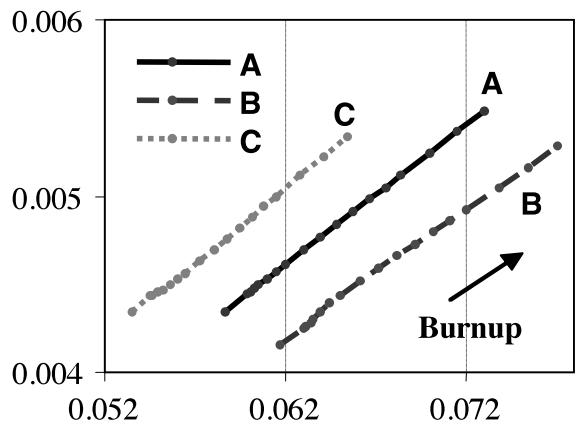

(b) $\Phi_{1}\left(\mathbf{n} / \mathrm{cm}^{2} \mathbf{s}\right)$

Fig. 8. Evolution with burnup of the fast group current-to-flux ratio for A, B, and C loading patterns in (a) a plane interface and (b) a corner interface. 

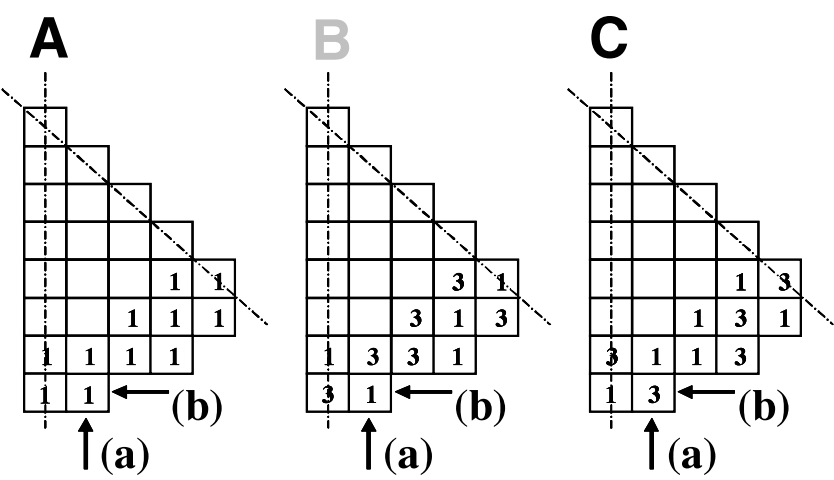

Fig. 9. Core loading patterns for standard PWR detailed 2-D calculations (numbers 1, 2, and 3 indicate fuel assemblies of different reactivities).

are found in the corner interfaces, in particular, between the $\mathrm{B}$ and $\mathrm{C}$ patterns, where the difference in fuel assembly reactivity is largest. The different neighborhood drives changes in the spectrum of the neutron leakage and thus in the reflector response through the reentrant core interfaces.

We summarize below our conclusions, extendible to the multigroup and 3-D cases:

1. Two-dimensional effects are observed at all interfaces (at both plane and corner interfaces).

2. The response matrices $[\beta]$ depend on the core loading pattern.

3. The cause is the neighbor effects that drive changes in transverse leakage: The surface flux is a consequence not only of the normal currents but also of the transverse currents.

Next, we derive a new method independent of the specific core loading. The response matrix at the core-reflector interface is decomposed into a response matrix in the absence of transverse leakage $\left[\beta_{0}\right]$ (corresponding to a $1-\mathrm{D}$ reflector case and then nondependent on the core configuration) and a flux vector induced by the transverse leakage (and then dependent on the core configuration):

$$
\begin{aligned}
\left.\mid \boldsymbol{\phi}_{g}(\text { interface })\right\rangle= & {\left.[\beta] \mid \boldsymbol{J}_{g}(\text { interface })\right\rangle } \\
= & {\left.\left[\beta_{0}\right] \mid \boldsymbol{J}_{g}(\text { interface })\right\rangle } \\
& \left.+\mid \boldsymbol{F}^{T}(\text { interface })\right\rangle .
\end{aligned}
$$

Then, the induced flux vector at the core-reflector interface can be written as a transverse response matrix and a vector of transverse leakage (difference on transverse currents). The transverse currents at the core-reflector interfaces are already available from the quadratic or cubic fit used in the nonlinear scheme (as explained at the end of Sec. III.A). Since only the fast transverse currents will be affected, the matrix elements corresponding to the thermal-to-fast current contributions are zero:

$$
\left.\left.\mid \boldsymbol{F}^{T}(\text { interface })\right\rangle=\left[\beta_{T}\right] \mid \Delta \boldsymbol{J}_{g}^{T}(\text { interface })\right\rangle .
$$

Equation (34) can be rewritten as follows, where the matrix in brackets is the multidimensional response matrix; note that when the transverse currents vanish, the response matrix reduces to the 1-D one:

$$
\begin{aligned}
\left.\mid \boldsymbol{\phi}_{g}(\text { interface })\right\rangle= & \left\{\left[\beta_{0}\right]+\left[\beta_{T}\right]\left|\Delta \boldsymbol{J}_{g}^{T}\right\rangle\left\langle\boldsymbol{J}_{g}\right|\right\} \\
& \left.\times \mid \boldsymbol{J}_{g}(\text { interface })\right\rangle .
\end{aligned}
$$

The 1-D response matrix $\left[\beta_{0}\right]$ can be analytically calculated, whereas $\left[\beta_{T}\right]$ has been determined by the aforementioned reference fine-mesh calculations, with results in Figs. 8 and 9. The transverse matrix $\left[\beta_{T}\right]$ obtained for those three reload patterns were nearly the same, so that we can conclude the following: (a) the transverse currents are responsible of the reflector response changes when changing the neighborhood and (b) the transverse matrix only needs to be calculated once for all reactors and cycles with the same reflector layout.

The change in the reflector response matrix with the change in reflector (downcomer) water density should be taken into account, but the change with the boron concentration is a minor one.

The above analytical method provides explicitly the reflector full response matrices for any structural material configuration and operating condition and thus allows for a more complete and accurate analysis of the relevant effects, as well as for the adequate correlation of the dependencies with nonlinear (core flux) effects.

\section{V.D. Validation of the Two-Dimensional Reflector Response Matrix Model in Two Groups of Energy}

Next, the validity of the reflector response matrix model is checked. The case under study is the initial core-loading pattern of the Spanish Ascó-II nuclear power plant (NPP), which is a Westinghouse-type PWR rated at $2775 \mathrm{MW}$ (thermal). As can be seen in Fig. 10, this pattern includes seven different types of fuel assemblies, identified by the enrichment and the number of burnable absorber pins. Six of these types are arranged in a checkerboard loading scheme, whereas the fuel assemblies of the highest reactivity are located at the core periphery. A steel shroud and a water reflector zone surround the core.

Comparisons were made between the results obtained with the 2-D fine-mesh transport-corrected diffusion COBAYA code, ${ }^{14}$ where the reflector is explicitly taken into account, and those obtained with the 2-D nodal SIMULA2D code, where the response matrices are used to obtain the boundary conditions in the core surroundings. Errors in power distributions due to the model implementation for the first cycle of the Ascó-II NPP are shown in Table V. 
TABLE V

Errors in Power Distributions

\begin{tabular}{|c|c|c|c|c|}
\hline $\begin{array}{l}\text { Burnup }(\mathrm{GWd} / \mathrm{t} \mathrm{U}) \\
\text { Power and Xenon }\end{array}$ & $\begin{array}{l}0.0 \\
\text { Hot Zero Power } \\
\text { Without Xenon }\end{array}$ & $\begin{array}{l}\quad 0.150 \\
\text { Hot Full Power } \\
\text { Equilibrium Xe }\end{array}$ & $\begin{array}{l}\quad 4.0 \\
\text { Hot Full Power } \\
\text { Equilibrium Xe }\end{array}$ & $\begin{array}{l}12.0 \\
\text { Hot Full Power } \\
\text { Equilibrium Xe }\end{array}$ \\
\hline $\begin{array}{l}\text { Mean deviation (\%) } \\
\text { Maximum deviation (\%) }\end{array}$ & $\begin{array}{r}0.06 \\
-0.22\end{array}$ & $\begin{array}{l}0.10 \\
0.49\end{array}$ & $\begin{array}{r}0.07 \\
-0.32\end{array}$ & $\begin{array}{l}0.09 \\
0.41\end{array}$ \\
\hline
\end{tabular}

We note that there is a quite good agreement with the reference case, from the beginning until the end of the cycle, with a mean error of $0.1 \%$ and a maximum error of $\sim 0.4 \%$.

\section{CONCLUSIONS}

We have developed and demonstrated the ACMFD method for multigroup - with any number of groupsand multidimensional diffusion calculations, including the consistent treatment of heterogeneous nodes in realistic fuel and core designs. The numerical and graphical analysis of the multigroup matrices and their eigenspectrum shows that complex eigenvalues and eigenfunctions (modes) appear frequently for more than two groups. Hence, the successful, efficient, and accurate extension of the ACMFD method to the complex modal space in 1-D to 3-D multigroup diffusion is one key achievement of this work.

We have also developed and demonstrated a new method for the calculation of multigroup response matrices at the core-reflector boundaries that, through the use of the ACMFD coupling equations, provides quite accurate power distributions in the active core region without explicit reflector nodes, as shown in realistic applications. These response matrices include the effects
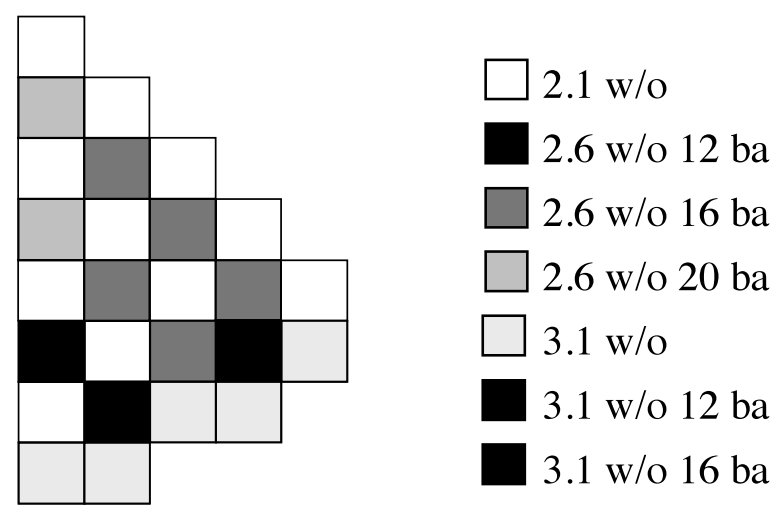

Fig. 10. Loading pattern in the first cycle of Ascó-II NPP. of heterogeneous reflectors and transverse (or reentrant) leakage in two or three dimensions, and are computed by detailed high-order reference calculations for use in 2-D or 3-D nodal calculations, independently of the core reload pattern, for all reactors with a similar reflector layout.

Ongoing work addresses the implementation of those methodologies and further validation, for an extended set of international benchmarks.

\section{ACKNOWLEDGMENTS}

This work is partially funded by the European Community Commission under the Sixth EURATOM Framework Programme, within the Integrated Project NURESIM "European Platform for Nuclear Reactor Simulations," contract 516560 (FI6O). It is also funded by the Ministerio de Ciencia y Tecnología of Spain within the program ENE2005-02064/ FTN, the Universidad Politécnica de Madrid and the Nuclear Association of Ascó and Vandellós-II power plants.

\section{REFERENCES}

1. Y. A. CHAO, "A Theoretical Analysis of the Coarse Mesh Finite Difference Representation in Advanced Nodal Methods," Mathematics and Computation, Reactor Physics and Environmental Analysis in Nuclear Applications, Vol. 1, p. 117, J. M. ARAGONÉS, Ed., Senda Ed., Madrid, Spain (1999).

2. Y. A. CHAO, "Comments on the Incompleteness of Analytic Base Function Expansion to Nodal Diffusion Equation Solution," Nucl. Sci. Eng., 147, 323 (2004).

3. C. AHNERT, J. M. ARAGONÉS, O. CABELLOS, and N. GARCÍA-HERRANZ, "Continuous Validation and Development for Extended Applications of the SEANAP Integrated 3-D PWR Core Analysis System," Mathematics and Computation, Reactor Physics and Environmental Analysis in Nuclear Applications, Vol. 1, p. 710, J. M. ARAGONÉS, Ed., Senda Ed., Madrid, Spain (1999).

4. N. GARCÍA-HERRANZ, J. M. ARAGONÉS, O. CABELLOS, and C. AHNERT, "Dependence of the Nodal Homogenized Two-Group Cross Sections on Intranodal Flux-Spectrum, Burnup and History," Mathematics and Computation, Reactor Physics and Environmental Analysis in Nuclear Applications, 
Vol. 1, p. 127, J. M. ARAGONÉS, Ed., Senda Ed., Madrid, Spain (1999).

5. N. GARCÍA-HERRANZ, O. CABELLOS, J. M. ARAGONÉS, and C. AHNERT, "Performance of the Analytic Coarse Mesh Finite Difference Method with Heterogeneous Nodes," Proc. Int. Conf. Reactor Physics (Physor-2002), Seoul, Korea, 2002, Vol. 1, p. 234, N. Z. CHO, Ed. (2002).

6. N. GARCÍA-HERRANZ, O. CABELLOS, J. M. ARAGONÉS, and C. AHNERT, "Analytic Coarse-Mesh FiniteDifference Method Generalized for Heterogeneous Multidimensional Two-Group Diffusion Calculations," Nucl. Sci. Eng., 144, 23 (2003).

7. R. S. VARGA, Matrix Iterative Analysis, Prentice-Hall, Englewood Cliffs, New Jersey (1962).

8. D. M. YOUNG, Iterative Solution of Large Linear Systems, Academic Press, New York (1971).

9. C. D. MEYER, Matrix Analysis and Applied Linear Algebra, Society for Industrial and Applied Mathematics, Philadelphia, Pennsylvania (2000).

10. Y. SAAD, Iterative Analysis for Sparse Linear Systems, 2nd ed., Society for Industrial and Applied Mathematics, Philadelphia, Pennsylvania (2003).

11. E. ANDERSON et al., LAPACK User's Guide, 3rd ed., Society for Industrial and Applied Mathematics, Philadelphia, Pennsylvania (1999).

12. A. F. HENRY, Nuclear Reactor Analysis, MIT Press, Cambridge, Massachusetts (1975).

13. C. AHNERT, "Desarrollo y Validación de Métodos de Cálculo de Núcleos Heterogeneos de Reactores de Agua Ligera," PhD Thesis, Universidad Complutense de Madrid (1985).

14. J. M. ARAGONÉS and C. AHNERT, "A Linear Discontinuous Finite Difference Formulation for Synthetic Coarse-
Mesh Few-Group Diffusion Calculations," Nucl. Sci. Eng., 94, 309 (1986).

15. Y. A. CHAO, "Coarse Mesh Finite Difference Methods and Applications," Proc. Int. Topl. Mtg. Advances in Reactor Physics and Mathematics and Computation into the Next Millennium (PHYSOR-2000), Pittsburgh, Pennsylvania, May 7-12, 2000, Vol. 1, p. 9404, American Nuclear Society (2000) (CD-ROM).

16. J. M. NOH and N. Z. CHO, "A New Approach of Analytic Basis Function Expansion to Neutron Diffusion Nodal Calculation," Nucl. Sci. Eng., 116, 165 (1994).

17. N. Z. CHO, Y. H. KIM, and K. W. PARK, "Extension of Analytic Function Expansion Nodal Method to Multigroup Problems in Hexagonal-Z Geometry," Nucl. Sci. Eng., 126, 35 (1997).

18. S. W. WOO, N. Z. CHO, and J. M. NOH, "The Analytic Function Expansion Nodal Method Refined with Transverse Gradient Basis Functions and Interface Flux Moments," Nucl. Sci. Eng., 139, 156 (2001).

19. K. KOEBKE, "Advances in Homogenization and Dehomogenization," Mathematical Methods for the Solution of Nuclear Engineering Problems, Vol. 2, p. 59, Fachinformationszentrum Energie, Physik, Mathematik GmbH, Munich, Germany (1981).

20. H. FINNEMANN, F. BENNEWITZ, and M. R. WAGNER, "Interface Current Techniques for Multi-Dimensional Reactor Calculations, Atomkernenergie, 30, 123 (1977).

21. M. R. WAGNER, K. KOEBKE, and H. J. WINTER, "A Non-Linear Extension of the Nodal Expansion Method," Mathematical Methods for the Solution of Nuclear Engineering Problems, Vol. 2, p. 43, Fachinformationszentrum Energie, Physik, Mathematik GmbH, Munich, Germany (1981).

22. J. R. ASKEW et al., J. Brit. Nucl. Energy Soc., 5, 564 (1966). 